\title{
A Novel Beamformer Robust to Steering Vector Mismatch
}

\author{
Chun-Yang Chen and P. P. Vaidyanathan \\ Dept. of Electrical Engineering, MC 136-93 \\ California Institute of Technology, Pasadena, CA 91125, USA \\ E-mail: cyc@caltech.edu,ppvnath@systems.caltech.edu
}

\begin{abstract}
It is well know that the performance of the minimum variance distortionless response (MVDR) beamformer is very sensitive to steering vector mismatch because of the signal cancellation. Such mismatches can occur as a result of direction-of arrival (DOA) errors, local scattering, near-far spatial signature mismatch, waveform distortion, source spreading, imperfectly calibrated arrays and distorted antenna shape.

In this paper, an adaptive beamformer which is robust against the DOA mismatch is proposed. This method imposes two quadratic constraints such that the magnitude responses of two steering vectors exceed unity. Then a diagonal loading method is used to force the magnitude responses within a range of the arrival angles to exceed unity. The diagonal loading factor can be computed systematically by a proposed algorithm. Numerical examples show that this method has a significantly better SINR performance compared to previously published methods (including the MVDR), and a complexity comparable to the standard MVDR beamformer.
\end{abstract}

\section{INTRODUCTION}

Beamforming has long been used in many areas, such as radar, sonar, seismology, medical imaging, speech processing and wireless communications. Introduction to beamforming can be found in [18]-[20] and the references therein. A datadependent beamformer was proposed by Capon in [1]. By exploiting the second order statistics of the array output, the method constrains the response of the signal of interest (SOI) to be unity and minimizes the total variance of the beamformer output. This method is called minimum variance distortionless response (MVDR) beamformer in the literature. The MVDR beamformer has very good resolution, and the SINR (signal-to-interference-plus-noise ratio) performance is much better than traditional data-independent beamformers. However, the steering vector of the SOI can be imprecise. When the mismatched steering vector is used in the MVDR beamformer, the response of the SOI is no longer constrained to be unity and thus is attenuated by the MVDR beamformer while minimizing the total variance of the beamformer output [2]. This phenomenon is called signal cancellation or selfnulling. It will dramatically degrade the output SINR. Many approaches, including [3]-[17] and the references therein, have been proposed for improving the robustness of the MVDR beamformer. A good introduction to this topic can be found in [5].

Recently, some approaches based on an uncertainty set of steering vectors have been proposed [4]-[9]. Instead of imposing a linear constraint, these approaches minimize the output variances subject to the constraint that the magnitude responses of a set of steering vectors exceed unity. If the mismatched steering vector is still in this set, its magnitude response will exceed unity and will not be attenuated. In this paper, we focus on the steering mismatch caused by direction-of-arrival (DOA) mismatch. Inspired by these uncertainty based methods, we

${ }^{1}$ Work supported in parts by the ONR grant N00014-06-1-0011, and the California Institute of Technology. consider a simplified uncertainty set which contains only the steering vectors with a desired uncertainty range of DOA. To find a suboptimal solution for this problem, the constraint is first loosened to two non-convex quadratic constraints such that the magnitude responses of two steering vectors exceed unity. Then a diagonal loading method is used to force the magnitude responses at the arrival angles between these two steering vectors to exceed unity. Therefore this method can always force the gains at a desired range of angles to exceed a constant level while suppressing the interferences and noise. A closed form solution to the proposed minimization problem is introduced, and the diagonal loading factor can be computed systematically by a proposed algorithm. Numerical examples show that this method has an excellent SINR performance and a complexity comparable to the standard MVDR beamformer (which has poor performance).

The rest of the paper is organized as follows. The analysis of steering vector mismatch and some previous work are reviewed in Section II. In Section III, we develop the theory and the algorithm of our new robust beamformer. The numerical examples are presented in Section IV. Section V gives the conclusions.

Notations. Bold faced lower case letters such as $\mathbf{y}$ represent vectors, and bold faced upper case letters such as $\mathbf{R}_{\mathbf{y}}$ denote matrices. The element in row $n$ and column $m$ of matrix $\mathbf{R}_{\mathbf{y}}$ is denoted by $R_{y, n, m}$. The notation $\mathbf{y}^{\dagger}$ denotes the conjugate transpose of the vector $\mathbf{y}$. The notation $E[x]$ denotes the expectation of the random variable $x$. The notation $W(z)$ denotes the z-transform of the elements in vector $\mathbf{w}$.

\section{The Stefring Vector Mismatch AND Some. PREVIOUS WORK}

Consider a uniform linear array (ULA) of $N$ omnidirectional sensors with interelement spacing $d$. The signal of interest (SOI) is a narrowband plane wave impinging from angle $\theta$ as shown in Fig. 1. The baseband array output $\mathbf{y}(t)$

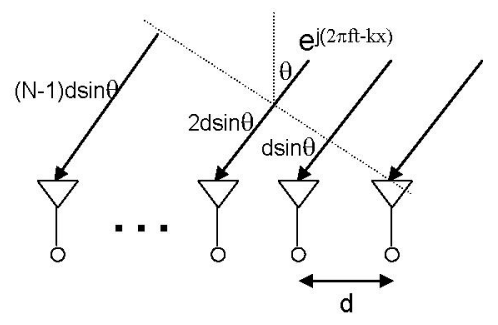

Fig. 1. The N-element uniform linear array.

can be expressed as

$$
\mathbf{y}(t)=x(t) \mathbf{s}(\theta)+\mathbf{v}(t)
$$


where $\mathbf{v}(t)$ denotes the sum of the interferences and the noises, $x(t)$ is the signal of interest (SOI), and $\mathbf{s}(\theta)$ represents the baseband array response of the SOI. It is called steering vector and can be expressed as

$$
\mathbf{s}(\theta) \triangleq\left(\begin{array}{llll}
1 & e^{j \frac{2 \pi}{\lambda} d \sin \theta} & \cdots & e^{j(N-1) \frac{2 \pi}{\lambda} d \sin \theta}
\end{array}\right)^{T}
$$

where $\lambda$ is the operating wavelength. The output of the beamformer can be expressed as $\mathbf{w}^{\dagger} \mathbf{y}(t)$, where $\mathbf{w}$ is the complex weighting vector. The output SINR (signal-to-interferencesplus-noise ratio) of the beamformer is defined as

$$
\mathrm{SINR} \triangleq \frac{E\left|x(t) \mathbf{w}^{\dagger} \mathbf{s}(\theta)\right|^{2}}{E\left|\mathbf{w}^{\dagger} \mathbf{v}(t)\right|^{2}}=\frac{\sigma_{x}^{2}\left|\mathbf{w}^{\dagger} \mathbf{s}(\theta)\right|^{2}}{\mathbf{w}^{\dagger} \mathbf{R}_{\mathbf{v}} \mathbf{w}}
$$

where $\mathbf{R}_{\mathbf{v}} \triangleq E\left[\mathbf{v}(t) \mathbf{v}^{\dagger}(t)\right]$, and $\sigma_{x}^{2} \triangleq E\left[|x(t)|^{2}\right]$. By exploiting the second order statistics of the array output $\mathbf{y}(t)$, the output SINR can be maximized by minimizing the total output variance while constraining the SOI response to be unity. This can be written as the following optimization problem:

$$
\begin{aligned}
& \min _{\mathbf{w}} \mathbf{w}^{\dagger} \mathbf{R}_{\mathbf{y}} \mathbf{w} \\
& \text { subject to } \mathbf{s}^{\dagger}(\theta) \mathbf{w}=1,
\end{aligned}
$$

where $\mathbf{R}_{\mathbf{y}} \triangleq E\left[\mathbf{y}(t) \mathbf{y}^{\dagger}(t)\right]$. The solution to this problem is well-known and was first given by Capon in [1] as

$$
\mathbf{w}_{c}=\frac{\mathbf{R}_{\mathbf{y}}^{-1} \mathbf{s}(\theta)}{\mathbf{s}^{\dagger}(\theta) \mathbf{R}_{\mathbf{y}}^{-1} \mathbf{s}(\theta)} .
$$

When there is a mismatch between the actual arrival angle $\theta$ and the assumed arrival angle $\theta_{m}$, this beamformer becomes

$$
\mathbf{w}_{m}=\frac{\mathbf{R}_{\mathbf{y}}{ }^{-1} \mathbf{s}\left(\theta_{m}\right)}{\mathbf{s}^{\dagger}\left(\theta_{m}\right) \mathbf{R}_{\mathbf{y}}{ }^{-1} \mathbf{s}\left(\theta_{m}\right)} .
$$

It can be viewed as the solution to the minimization problem

$$
\begin{aligned}
& \min _{\mathbf{w}} \mathbf{w}^{\dagger} \mathbf{R}_{\mathbf{y}} \mathbf{w} \\
& \text { subject to } \mathbf{s}^{\dagger}\left(\theta_{m}\right) \mathbf{w}=1 .
\end{aligned}
$$

Because $\mathbf{w}^{\dagger} \mathbf{R}_{\mathbf{y}} \mathbf{w}=\mathbf{w}^{\dagger} \mathbf{R}_{\mathbf{v}} \mathbf{w}+\sigma_{x}^{2}\left|\mathbf{s}^{\dagger}(\theta) \mathbf{w}\right|^{2}$, and $\mathbf{s}^{\dagger}(\theta) \mathbf{w}=1$ is no longer valid due to the mismatch, the SOI is interpreted as an interference. The SOI magnitude response is thus suppressed as a part of the objective function. When the SOI is strong, the beamformer tends to place a zero around the SOI arrival angle $\theta$ to minimize the total output variance. This will almost destroy the SOI response. A small mismatch could cause a severe degradation in the SINR.

Many approaches have been proposed for improving the robustness of the standard MVDR beamformer during the past three decades. In [10], the following modification of $\mathbf{R}_{\mathbf{y}}$ is performed before obtaining the MVDR beamformer by Fq. (3):

$$
\mathbf{R}_{\mathbf{y}} \leftarrow \mathbf{R}_{\mathbf{y}}+\gamma \mathbf{I}_{\mathbf{N}}
$$

This approach is called diagonal loading in the literature. It increases the variance of the artificial white noise by $\gamma$ in $\mathbf{R}_{\mathbf{y}}$. This modification forces the beamformer to put more effort on suppressing white noise rather than interferences. When the SOI steering vector is mismatched, the SOI is attenuated as one of the interferences. As the beamformer puts less effort on suppressing the interferences, the SOI suppression problem addressed above is reduced. However, when $\gamma$ is too large, the beamformer fails to suppress strong interferences because it puts most effort to suppress the white noise. There is a trade-off between reducing SOI suppression and suppressing interferences. It is not clear how to choose a good diagonal loading factor $\gamma$ in the traditional diagonal loading methods.

In [4], the following optimization problem is considered:

$$
\begin{aligned}
& \min _{\mathbf{w}} \mathbf{w}^{\dagger} \mathbf{R}_{\mathbf{y}} \mathbf{w} \\
& \text { subject to }\left|\mathbf{w}^{\dagger} \mathbf{s}\right| \geq 1, \forall \mathbf{s} \in \mathcal{E},
\end{aligned}
$$

where $\mathcal{E}$ is a sphere defined as

$$
\mathcal{E}=\{\overline{\mathbf{s}}+\mathbf{e} \mid\|\mathbf{e}\| \leq 1\},
$$

where $\overline{\mathbf{s}}$ is the assumed steering vector. Instead of imposing a linear constraint, this method constrains the magnitude responses of a sphere set of steering vectors to exceed unity. If the mismatched steering vector is in this uncertainty set, its gain will exceed unity and will not be attenuated. Unlike the method in [10], this method directly protects the SOI response from being suppressed. In [5], [6], the uncertainty set in Eq. (6) has been generalized to an ellipsoid. This method takes into account any steering vector mismatch that keeps the vector in the sphere in Eq. (6). If the primary mismatch is known to be angle of arrival mismatch, then the set in Eq. (6) can be replaced with a much smaller set, thereby resulting in better performance. In this paper, we take such an approach.

\section{NEW ROBUST BEAMFORMER}

In this paper, we focus on the direction-of-arrival (DOA) mismatch. When there is a DOA mismatch, the minimization in Eq. (5) suppresses the magnitude response of the actual SOI. To avoid this, we should force the magnitude responses at a range of arrival angles to exceed unity while minimizing the total output variance. This optimal robust beamformer problem can be expressed as

$$
\begin{aligned}
& \mathbf{w}_{d}=\arg \min _{\mathbf{w}} \mathbf{w}^{\dagger} \mathbf{R}_{\mathbf{y}} \mathbf{w} \\
& \text { subject to }\left|\mathbf{s}^{\dagger}(\theta) \mathbf{w}\right| \geq 1 \text { for } \theta_{1} \leq \theta \leq \theta_{2},
\end{aligned}
$$

where $\theta_{1}$ and $\theta_{2}$ are the lower and upper bounds of the SOI arrival angle respectively, and $\mathbf{s}(\theta)$ is the steering vector defined in Fq. (1) with the arrival angle $\theta$. The following uncertainty set of steering vectors is considered:

$$
\left\{\mathbf{s}=\left(\begin{array}{cccc}
1 & e^{j \omega} & \cdots & e^{j(N-1) \omega}
\end{array}\right)^{T} \mid \omega_{1} \leq \omega \leq \omega_{2}\right\},
$$

where $\omega_{1} \triangleq 2 \pi \sin \theta_{1} / \lambda$, and $\omega_{2} \triangleq 2 \pi \sin \theta_{2} / \lambda$. Unlike other uncertainty set based approaches using ellipsoid, spherical, or polyhedron sets, the uncertainty set we considered in Fq. (8) is a curve. This constraint protects the signals in the range of angles $\theta_{1} \leq \theta \leq \theta_{2}$ from being suppressed.

\section{A. Two-point quadratic constraint}

There is no standard tool for solving the optimal beamformer $\mathbf{w}_{d}$ in $\mathrm{Eq}$. (7) because the constraint does not fit any of the existing standard optimization tools. The constraint $\left|\mathbf{s}^{\dagger}(\theta) \mathbf{w}\right|^{2} \geq 1$ for $\theta_{1} \leq \theta \leq \theta_{2}$ can be viewed as infinite number of quadratic constraints. Other uncertainty steering vector based methods solve this problem by adding more constraints so that the problem fits some standard optimization tools such as SOCP. Our approach does this in an opposite way. We start looking for the solution by loosening the constraint. We first loosen the constraint by choosing only two constraints $\left|\mathbf{s}^{\dagger}\left(\theta_{1}\right) \mathbf{w}\right|^{2} \geq 1$ and $\left|\mathbf{s}^{\dagger}\left(\theta_{2}\right) \mathbf{w}\right|^{2} \geq 1$ from 
the infinite constraints $\left|\mathbf{s}^{\dagger}(\theta) \mathbf{w}\right|^{2} \geq 1$ for $\theta_{1} \leq \theta \leq \theta_{2}$. The corresponding optimization problem can be written as

$$
\begin{aligned}
& \mathbf{w}_{0}=\arg \min _{\mathbf{w}} \mathbf{w}^{\dagger} \mathbf{R}_{\mathbf{y}} \mathbf{w} \\
& \text { subject to }\left|\mathbf{s}^{\dagger}\left(\theta_{1}\right) \mathbf{w}\right| \geq 1, \text { and }\left|\mathbf{s}^{\dagger}\left(\theta_{2}\right) \mathbf{w}\right| \geq 1 .
\end{aligned}
$$

Because the constraint is loosened, the minimum to this problem is a lower bound of the original problem in Fq. (7). In order to obtain an analytic solution, we recast the problem to the following equivalent form:

$$
\begin{aligned}
& \min _{\mathbf{w}, \phi, \rho_{0} \geq 1, \rho_{1} \geq 1} \mathbf{w}^{\dagger} \mathbf{R}_{\mathbf{y}} \mathbf{w} \\
& \text { subject to } \mathbf{S}^{\dagger} \mathbf{w}=\left(\begin{array}{c}
\rho_{0} \\
\rho_{1} e^{j \phi}
\end{array}\right),
\end{aligned}
$$

where $\mathbf{S}=\left(\begin{array}{ll}\mathbf{s}\left(\theta_{1}\right) & \mathbf{s}\left(\theta_{2}\right)\end{array}\right)$, and $\rho_{0}, \rho_{1}$ and $\phi$ are real numbers.

To solve this problem, we first assume $\phi, \rho_{0}$ and $\rho_{1}$ are given and solve $\mathbf{w}$. The solution $\mathbf{w}_{0}$ can be obtained by Lagrange multiplier method and can be expressed as

$$
\mathbf{w}_{0}=\mathbf{R}_{\mathbf{y}}{ }^{-1} \mathbf{S}\left(\mathbf{S}^{\dagger} \mathbf{R}_{\mathbf{y}}{ }^{-1} \mathbf{S}\right)^{-1}\left(\begin{array}{c}
\rho_{0} \\
\rho_{1} e^{j \phi}
\end{array}\right) .
$$

Given $\phi, \rho_{0}$ and $\rho_{1}, \mathbf{w}_{0}$ can be found from the above equation. The task now is to solve for $\phi, \rho_{0}$ and $\rho_{1}$. Write

$$
\left(\mathbf{S}^{\dagger} \mathbf{R}_{\mathbf{y}}{ }^{-1} \mathbf{S}\right)^{-1}=\left(\begin{array}{cc}
r_{0} & r_{2} e^{j \beta} \\
r_{2} e^{-j \beta} & r_{1}
\end{array}\right)
$$

where $r_{0}, r_{1}$ and $r_{2}$ are real nonnegative numbers. Substituting $\mathbf{w}_{0}$ in $\mathrm{Fq}$. (10) into the objective function, it becomes

$$
\begin{aligned}
\mathbf{w}_{0}^{\dagger} \mathbf{R}_{\mathbf{y}} \mathbf{w}_{0} & =\left(\begin{array}{ll}
\rho_{0} & \rho_{1} e^{-j \phi}
\end{array}\right)\left(\mathbf{S}^{\dagger} \mathbf{R}_{\mathbf{y}}{ }^{-1} \mathbf{S}\right)^{-1}\left(\begin{array}{c}
\rho_{0} \\
\rho_{1} e^{j \phi}
\end{array}\right) \\
& =r_{0} \rho_{0}^{2}+r_{1} \rho_{1}^{2}+2 \operatorname{Re}\left\{r_{2} \rho_{0} \rho_{1} e^{j(\beta+\phi)}\right\} \\
& \geq r_{0} \rho_{0}^{2}+r_{1} \rho_{1}^{2}-2 r_{2} \rho_{0} \rho_{1} .
\end{aligned}
$$

To minimize the objective function, $\phi$ can be chosen as

$$
\phi=-\beta+\pi
$$

so that the last equality in Fq. (11) holds. Now $\phi$ and $\mathbf{w}_{0}$ are obtained by Eq. (12) and Fq. (10), and the objective function becomes Fq. (11). To further minimize the objective function, $\rho_{0}$ and $\rho_{1}$ can be found by solving the following optimization problem:

$$
\min _{\rho_{0} \geq 1, \rho_{1} \geq 1} r_{0} \rho_{0}^{2}+r_{1} \rho_{1}^{2}-2 r_{2} \rho_{0} \rho_{1} .
$$

This can be solved by using the Karush-Kuhn-Tucker (KKT) condition. The following solution can be obtained:

$$
\begin{aligned}
& \rho_{0}=\left\{\begin{array}{ll}
1, & r_{2} / r_{0} \leq 1 \\
r_{2} / r_{0}, & r_{2} / r_{0}>1
\end{array},\right. \\
& \rho_{1}=\left\{\begin{array}{ll}
1, & r_{2} / r_{1} \leq 1 \\
r_{2} / r_{1}, & r_{2} / r_{1}>1
\end{array} .\right.
\end{aligned}
$$

Summarizing Eq. (12), Eq. (13) and Eq. (10), the following algorithm for solving the beamformer with the two-point quadratic constraint in Fq. (9) is obtained.
Algorithm 1: Given $\theta_{1}, \theta_{2}$ and $\mathbf{R}_{\mathbf{y}}, \mathbf{w}_{0}$ can be computed by the following steps:

$$
\begin{array}{ll}
\text { 1. } & \mathbf{S} \leftarrow\left(\mathbf{s}\left(\theta_{1}\right) \mathbf{s}\left(\theta_{2}\right)\right) . \\
\text { 2. } & \mathbf{V} \leftarrow\left(\mathbf{R}_{\mathbf{y}}\right)^{-1} \mathbf{S} . \\
\text { 3. } & \mathbf{R} \triangleq\left(\begin{array}{cc}
r_{0} & r_{2} e^{j \beta} \\
r_{2} e^{-j \beta} & r_{1}
\end{array}\right) \leftarrow\left(\mathbf{S}^{\dagger} \mathbf{V}\right)^{-1} . \\
\text { 4. } & \phi \leftarrow-\beta+\pi . \\
& \rho_{0} \leftarrow\left\{\begin{array}{ll}
1, & r_{2} / r_{0} \leq 1 \\
r_{2} / r_{0}, & r_{2} / r_{0}>1
\end{array} .\right. \\
& \rho_{1} \leftarrow\left\{\begin{array}{ll}
1, & r_{2} / r_{1} \leq 1 \\
r_{2} / r_{1}, & r_{2} / r_{1}>1
\end{array} .\right. \\
\text { 5. } & \mathbf{w}_{0} \leftarrow \mathbf{V R}\left(\begin{array}{c}
\rho_{0} \\
\rho_{1} e^{j \phi}
\end{array}\right) .
\end{array}
$$

The matrix inversion in Step 2 contains most of the complexity of the algorithm. Therefore the algorithm has the same order of complexity as the MVDR beamformer. Because the constraint is loosened, the feasible set of the two-point quadratic constraint problem in Eq. (9) is a superset of the feasible set of the original problem in Fq. (7). The minimum found in this problem is a lower bound of the minimum of the original problem. If the solution $\mathbf{w}_{0}$ in the two-point quadratic constraint problem in $\mathrm{Eq}$. (9) happens to satisfy the original constraint $\left|\mathbf{s}^{\dagger}(\theta) \mathbf{w}_{0}\right|^{2} \geq 1$ for $\theta_{1} \leq \theta \leq \theta_{2}$, then $\mathbf{w}_{0}$ is also the solution to the original problem in Fq. (7). Unfortunately, in general the original constraint $\left|\mathbf{s}^{\dagger}(\theta) \mathbf{w}\right| \geq 1$ for $\theta_{1} \leq \theta \leq \theta_{2}$ is not guaranteed to be satisfied by the solution to $\mathrm{Eq}$. (9). This problem will be overcome by a method provided in the next section.

\section{B. Two-point quadratic constraint with diagonal loading}

Substituting Eq. (8) into the constraint in Eq. (7), we can rewrite the robust condition into $\left|W\left(e^{j \omega}\right)\right| \geq 1$ for $\omega_{1} \leq \omega \leq$ $\omega_{2}$. If the robust condition is not satisfied, $W(z)$ must have a zero whose angle is between $\omega_{1}$ and $\omega_{2}$. If $\theta_{1}$ and $\theta_{2}$ are close, the zero is also close to both of the quadratic-constraint points. Thus, it attenuates the gain at these points. However, the magnitude responses at these points are constrained to exceed unity. To satisfy the constraints, the overall energy of $\mathbf{w}$ must be adjusted to a certain high level. Therefore, if the robust condition $\left|\mathbf{s}^{\dagger}(\theta) \mathbf{w}\right| \geq 1$ for $\theta_{1} \leq \theta \leq \theta_{2}$ is not satisfied, the norm of the weighting vector $\|w\|$ will become very large. By using this fact, we can impose some penalty on $\|\mathbf{w}\|^{2}$ to avoid this situation from happening. This can be done by the diagonal loading approach mentioned in Sec. II. The corresponding optimization problem can be written as

$$
\begin{aligned}
& \mathbf{w}_{\gamma}=\arg \min _{\mathbf{w}} \mathbf{w}^{\dagger} \mathbf{R}_{\mathbf{y}} \mathbf{w}+\gamma\|\mathbf{w}\|^{2} \\
& \text { subject to }\left|\mathbf{s}^{\dagger}\left(\theta_{1}\right) \mathbf{w}\right| \geq 1, \text { and }\left|\mathbf{s}^{\dagger}\left(\theta_{2}\right) \mathbf{w}\right| \geq 1,
\end{aligned}
$$

where $\gamma$ is the diagonal loading factor which represents the amount of the penalty put on $\|\mathbf{w}\|^{2}$. The solution $\mathbf{w}_{\gamma}$ can be found by performing the following modification on the output covariance matrix:

$$
\mathbf{R}_{\mathbf{y}} \leftarrow \mathbf{R}_{\mathbf{y}}+\gamma \mathbf{I}_{N}
$$

and then applying Algorithm 1. When $\gamma \rightarrow \infty$, the solution converges to

$$
\begin{aligned}
& \mathbf{w}_{\infty}=\arg \min _{\mathbf{w}}\|\mathbf{w}\|^{2} \\
& \text { subject to }\left|\mathbf{s}^{\dagger}\left(\theta_{1}\right) \mathbf{w}\right| \geq 1, \text { and }\left|\mathbf{s}^{\dagger}\left(\theta_{2}\right) \mathbf{w}\right| \geq 1 .
\end{aligned}
$$


The following lemma gives the condition for which $\mathbf{w}_{\infty}$ satisfies the constraint $\left|\mathbf{s}(\theta)^{\dagger} \mathbf{w}_{\infty}\right| \geq 1$ for all $\theta$ in $\theta_{1} \leq \theta \leq \theta_{2}$.

Lemma 1: $\left|\mathbf{s}^{\dagger}(\theta) \mathbf{w}_{\infty}\right| \geq 1$ for $\theta_{1} \leq \theta \leq \theta_{2}$ if and only if $\left|\sin \theta_{2}-\sin \theta_{1}\right| \leq \lambda /(d N)$.

Proof: According to Fq. (15), substituting $\mathbf{R}_{\mathbf{y}}=\mathbf{I}_{N}$ and applying Algorithm 1, one can obtain

$\mathbf{w}_{\infty}=\frac{1}{N+\left|\operatorname{sincd}\left(\frac{\omega_{2}-\omega_{1}}{2}\right)\right|}\left(\mathbf{s}\left(\theta_{1}\right)+\mathbf{s}\left(\theta_{2}\right) e^{j \frac{\left(\omega_{2}-\omega_{1}\right)(N-1)}{2}}\right)$,

where

$$
\begin{aligned}
& \omega_{1} \triangleq \frac{2 \pi}{\lambda} d \sin \theta_{1}, \omega_{2} \triangleq \frac{2 \pi}{\lambda} d \sin \theta_{2}, \text { and } \\
& \operatorname{sincd}(\omega) \triangleq \frac{\sin (\omega N)}{\sin \omega} .
\end{aligned}
$$

By direct substitution, one can obtain

$$
\left|\mathbf{s}^{\dagger}(\theta) \mathbf{w}_{\infty}\right|=\left|\frac{\operatorname{sincd}\left(\frac{\omega_{1}-\omega}{2}\right)+a \cdot \operatorname{sincd}\left(\frac{\omega_{2}-\omega}{2}\right)}{N+\left|\operatorname{sincd}\left(\frac{\omega_{2}-\omega_{1}}{2}\right)\right|}\right|,
$$

where $\omega \triangleq \frac{2 \pi}{\lambda} d \sin \theta$ and

$$
a= \begin{cases}1 & , \text { if } \operatorname{sincd}\left(\frac{\omega_{2}-\omega_{1}}{2}\right)>0 \\ -1 & , \text { otherwise. }\end{cases}
$$

By Eq. (16), it can be verified that

$$
\left|\mathbf{s}^{\dagger}(\theta) \mathbf{w}_{\infty}\right| \geq 1 \text { for } \omega_{1} \leq \omega \leq \omega_{2}
$$

if and only if

$$
\left|\omega_{2}-\omega_{1}\right| \leq \frac{2 \pi}{N}
$$

which can also be expressed as $\left|\sin \theta_{2}-\sin \theta_{1}\right| \leq \lambda /(d N)$.

If the condition $\left|\sin \theta_{1}-\sin \theta_{2}\right| \leq \lambda /(d N)$ is satisfied, there exists a $\gamma>0$ such that the condition $\left|\mathbf{s}^{\dagger}(\theta) \mathbf{w}_{\gamma}\right| \geq 1$ for $\theta_{1} \leq \theta \leq \theta_{2}$ is satisfied. However, introducing the diagonal loading changes the objective function $\mathbf{w}^{\dagger} \mathbf{R}_{\mathbf{y}} \mathbf{w}$ to $\mathbf{w}^{\dagger}\left(\mathbf{R}_{\mathbf{y}}+\gamma \mathbf{I}_{N}\right) \mathbf{w}$. The modification of the objective function affects the suppression of the interferences. To keep the objective function correct, $\gamma$ should be chosen as small as possible while the condition $\left|\mathbf{s}^{\dagger}(\theta) \mathbf{w}\right| \geq 1$ for $\theta_{1} \leq \theta \leq \theta_{2}$ is satisfied. For finding such a $\gamma$, we propose the following algorithm:

Algorithm 2: Given $\theta_{1}, \theta_{2}, \mathbf{R}_{\mathbf{y}}$, an initial value of $\gamma$, a search step size $\alpha>1$ and a set of angles, $\zeta_{i}, i=1,2, \cdots, n$ which satisfies $\theta_{1}<\zeta_{i}<\theta_{2}$ for all $i, \mathbf{w}_{\gamma}$ can be computed by the following steps:

1. $\mathbf{R}_{\mathbf{y}} \leftarrow \mathbf{R}_{\mathbf{y}}+\gamma \mathbf{I}_{N}$.

2. Compute $\mathbf{w}_{\gamma}$ by Algorithm 1 .

3. If $\left|\mathbf{s}^{\dagger}\left(\zeta_{i}\right) \mathbf{w}_{\gamma}\right| \geq 1$ for all $i=1,2, \cdots, n$ then stop. else $\gamma \leftarrow \alpha \gamma$, and go to 1 .

This algorithm keeps increasing $\gamma$ by multiplying $\alpha$ until $\left|\mathbf{s}^{\dagger}\left(\zeta_{i}\right) \mathbf{w}_{\gamma}\right| \geq 1$ for all $i=1,2, \cdots, n$ is satisfied. This is an approximation for $\left|\mathbf{s}^{\dagger}(\theta) \mathbf{w}_{\gamma}\right| \geq 1$ for $\theta_{1} \leq \theta \leq \theta_{2}$. In the numerical results in next section, the number $n$ can be very small. The choice $n=3$ works well in all of the examples. Also, the SINR is not sensitive to the choice of $\alpha$.

\section{NUMERICAI EXAMPI.ES}

For the purpose of design examples, the same parameters used in [6] are used in this section. An uniform linear array (ULA) of $N=10$ omnidirectional sensors spaced halfwavelength apart (i.e. $d=\lambda / 2$ ) is considered. There are three signals impinging upon this array:

1) the signal of interest (SOI) $x(t)$ with angle of arrival $\theta$,

2) an interference signal $x_{i n t 1}(t)$ with angle of arrival $\theta_{\text {int } 1}=30^{\circ}$ and $\sigma_{i n t 1}^{2}=10^{4}(40 \mathrm{~dB}$ above noise) and

$3)$ another interference signal $x_{i n t 2}(t)$ with angle of arrival $\theta_{\text {int } 2}=75^{\circ}$ and $\sigma_{\text {int } 2}^{2}=10^{2}(20 \mathrm{~dB}$ above noise $)$.

The received noise variance $\sigma^{2}=1$. The actual arrival angle $\theta$ is $43^{\circ}$ but the assumed arrival angle $\theta_{m}$ is $45^{\circ}$.

Example 1: SINR versus diagonal loading factors $\gamma$.

In this example, the SNR is 10dB. The SINR defined in Eq. (2) is compared for different diagonal loading factors $\gamma$. The following methods are considered:

1) Algorithm 7 with $\theta_{1}=42^{\circ}$ and $\theta_{2}=48^{\circ}$.

2) General-rank method [9] with the parameter

$$
\epsilon=\max _{48^{\circ} \geq \theta \geq 42^{\circ}}\left\|\mathbf{s}(\theta) \mathbf{s}^{\dagger}(\theta)-\mathbf{s}\left(45^{\circ}\right) \mathbf{s}^{\dagger}\left(45^{\circ}\right)\right\|_{F} \approx 4.73 .
$$

3) Diagonal loading method [10].

4) Directional LCMV (linear constrained minimum variance) [13] with two linear constraints which forces the responses of the signals from $42^{\circ}$ and $48^{\circ}$ to be unity.

5) Derivative $L C M V$ [15] with two linear constraints which forces the responses of the signals from $45^{\circ}$ to be unity and the derivative of the beampattern on $45^{\circ}$ to be zero.

One can verify that the choice of $\theta_{1}, \theta_{2}, d$ and $N$ satisfies $\left|\sin \theta_{1}-\sin \theta_{2}\right| \leq \lambda /(d N)$. Therefore, by Lemma 1 , there exist a $\gamma>0$ so that $\left|\mathbf{s}(\theta) \mathbf{w}_{\gamma}\right| \geq 1$ for $\theta_{1} \leq \theta \leq \theta_{2}$. The SINR of the MVDR beamformer without mismatch is also plotted. This is an upper bound on the SINR. Fig. 2 shows the result for $S N R=10 \mathrm{~dB}$. One can observe that there is

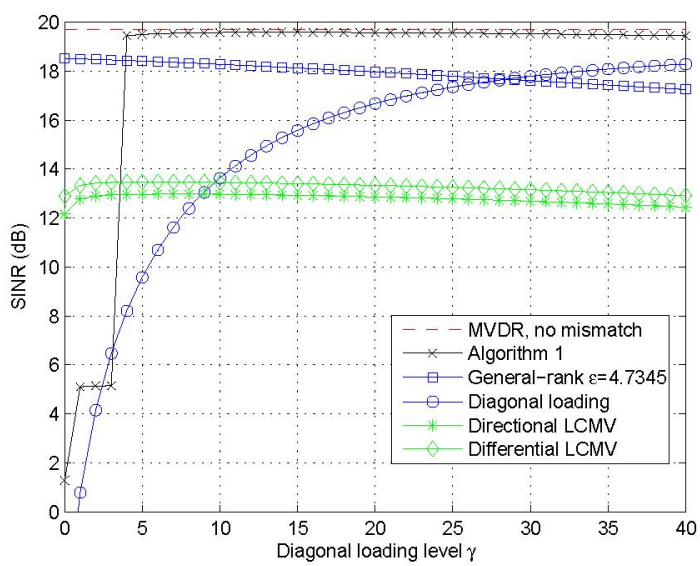

Fig. 2. Fxample 1: SINR versus $\gamma$ with SNR $=10 \mathrm{~dB}$.

a huge jump in the SINR of Algorithm 1 around $\gamma=3$. When this happens, the SINR of Algorithm 1 increases significantly and becomes very close to the upper bound provided by the MVDR beamformer without mismatch. This jump happens when the robust condition $\left|\mathbf{s}(\theta) \mathbf{w}_{\gamma}\right| \geq 1$ for $\theta_{1} \leq \theta \leq \theta_{2}$ is satisfied. After the jump, the SINR decays 
slowly as $\gamma$ increases because of the over-suppression of white noise. Observing Fig. 2, we can see why Algorithm 2 works. Algorithm 2 increases $\gamma$ by repeatedly multiplying $\alpha$ until $\mathbf{w}_{\gamma}$ satisfies $\left|\mathbf{w}_{\gamma}^{\dagger} \mathbf{s}\left(\zeta_{i}\right)\right| \geq 1$ for $i=1,2, \cdots, n$. This happens as $\gamma$ crosses the jump in SINR. Also, the SINR is not sensitive to the choice of $\alpha$ because the SINR decays very slow after the jump. By Algorithm 2, we can find a suitable $\gamma$ with only a few iterations. For other approaches involving diagonal loading, it is not clear how to find a good diagonal loading factor $\gamma$.

Example 2: SINR versus SNR.

In this example, the SINR in Fq. (2) are compared for different SNRs ranged from $-20 \mathrm{~dB}$ to $30 \mathrm{~dB}$. The following methods are considered:

1) Algorithm 2 with $\theta_{1}=42^{\circ}, \theta_{2}=48^{\circ}, \zeta_{1}=43.5^{\circ}$, $\zeta_{2}=45^{\circ}, \zeta_{3}=46.5^{\circ}$, initial $\gamma=1$ and step size $\alpha=2$.

2) General-rank method. Same as in Example 1.

3) Extended diagonal loading method [4]-[6] with the parameter

$$
\epsilon=\max _{48^{\circ} \geq \theta \geq 42^{\circ}}\left\|\mathrm{s}(\theta)-\mathrm{s}\left(45^{\circ}\right)\right\| \approx 1.95 .
$$

4) Directional LCMV [13], [14] with two linear constraints at the angles $42^{\circ}, 48^{\circ}$.

5) Directional LCMV with three linear constraints at the angles $42^{\circ}, 45^{\circ}$ and $48^{\circ}$.

6) Derivative $L C M V$ with two linear constraints [15]. Same as in Example 1.

7) Derivative LCMV with three linear constraints which force the responses of the signals from $45^{\circ}$ to be unity and both the first and second derivatives of the beampattern on $45^{\circ}$ to be zero.

8) The standard MVDR beamformer in Eq. (4).

Again, the SINR of the MVDR beamformer without mismatch is also plotted. It provides the upper bound on the SINR of these robust beamformers. The results are shown in Fig. 3. The SINR of the standard MVDR beamformer

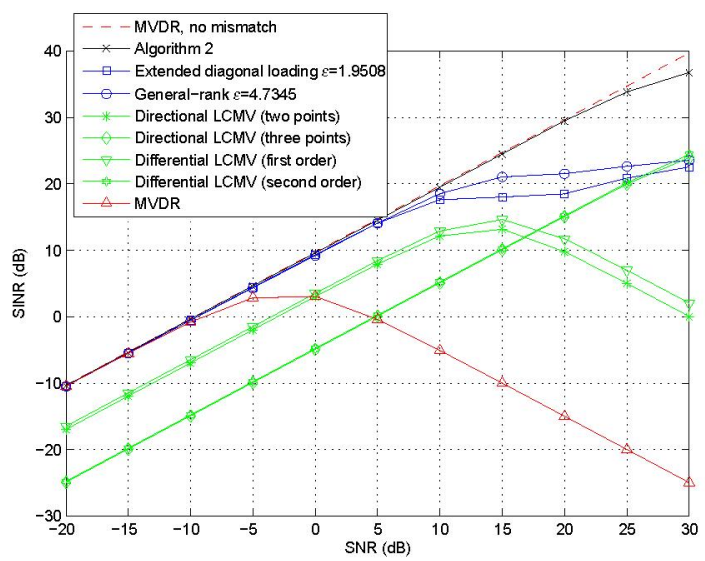

Fig. 3. Example 2: SINR versus SNR with $\gamma=10$.

is seriously degraded with only $2^{\circ}$ of mismatch. When the SNR increases, the MVDR beamformer tends to suppress the strong SOI to minimize the total output variance. Therefore, in the high SNR region, the SINR decreases when SNR increases. The performances of the uncertainty based methods are better than the LCMV methods. This is because the linear equality constraints are too strong compared to the quadratic inequality constraints. Among the uncertainty based methods, Algorithm 2 has the best SINR performance because it has an uncertainty set which focuses on the DOA mismatch only. The SINR is very close to the upper bound provided by the MVIR beamformer without mismatch.

\section{Conctusions}

In this paper, a new beamformer wich is robust against DOA mismatch is introduced. This robust beamformer quadratically constrains the magnitude responses of two steering vectors and then uses a diagonal loading method to force the magnitude response in a range of arrival angles to exceed unity. Therefore this method can always force the gains in a desired range of angles to exceed a constant level. The analytic solution to the quadratic constraint minimization problem has been proposed in Algorithm 1, and the diagonal loading factor $\gamma$ can be determined by a simple iteration method proposed in Algorithm 2. The complexity required in Algorithm 1 is approximately about the same as in the MVDR beamformer. The overall complexity depends on the number of iterations in Algorithm 2 which depends on the SNR. In our numerical examples, when SNR $<10 \mathrm{~dB}$, the number of iterations is less than three. The numerical examples also show that our approach has a significantly better SINR performance compared to previously published methods.

\section{REFERENCES}

[1] J. Capon, "High-resolution frequency-wavenumber spectrum analysis," Proc. IEEE, pp. 1408-1418, Aug. 1969.

[2] H. Cox, "Resolving power and sensitivity to mismatch of optimum array processors," J. Acoust. Soc. Amer. pp. 771-758, 1973

[3] J. I.i and P. Stoica, "Robust Adaptive Beamforming,", John Wiley \& Sons, 2006

[4] S. Vorobyov, A. B. Gershman, and 7.-Q. I.uo, "Robust adaptive beamforming using worst-case performance optimization: A solution to the signal mismatch problem," IEEE Trans. SP, pp. 313-324, Feb. 2003.

[5] J. I.i, P. Stoica, and 7. Wang, "On Robust Capon Beamforming and Diagonal I oading," IEEE Trans. SP, pp. 1702-1714, July 2003.

[6] R. G. I orenz and S. P. Boyd, "Robust Minimum Variance Beamforming," IEEE Trans. SP, pp. 1684-1696, May 2005.

[7] I. I.. Krolik, "The performance of matched-field beamformers with Mediterranean vertical array data,"IEEE Trans. SP. pp. 2605-2611.

[8] S. Q. Wu and J. Y. Zhang, "A new robust beamforming method with antennae calibration erros," in Proc. IEEE Wireless Commun. Networking Conf., New Orleans, I.A, Sept. 1999, pp. 869-872

[9] S. Shahbazpanahi, A. B. Gershman, 7..-Q. I.uo, and K. M. Wong, "Robust adaptive beamforming for general-rank signal models, "IEEE Trans. SP, pp. 2257-2269, Sept. 2003 .

[10] B. D. Carlson, "Covariance matrix estimation errors and diagonal loading in adaptive arrays," IEEE Trans. Aerosp. Electron. Syst., pp. 397 401, July 1988.

[11] K. I. Bell, Y. Ephraim, and H. I. Van Trees, "A Bayesian approach to robust adaptive beamforming," IFEE Trans. SP, pp. 386-398, Feb. 2000.

[12] J. R. Guerci, "Theory and Application of Covariance Matrix Tapers for Robust Adaptive Beamforming," IEEE Trans. SP, pp. 977-985, April 1999.

[13] A. H. Booker, C.Y. Ong, J.P. Burg, and G.D. Hair, Multiple-constraint adaptive filtering. Dallas, TX: Texas Instrum. Sci. Services Div., April 1969.

[14] O. I. Forst, III, "An algorithm for linearly constrained adaptive processing," Proc. IEEE, vol. 60, pp. 926-935, Aug. 1972.

[15] S. P. Applebaum and D. J. Chapman, "Adaptive arrays with main beam constraints," IEEE Trans. Ant. Prop., vol. AP-24, pp. 650-662, Sept. 1976.

[16] K. I.. Bell, Y. Ephraim, and H. I.. Van Trees, "A Bayesian approach to robust adaptive beanforming," IEEE Trans. Sig. Proc., vol. 48, pp. 386-398, Feb. 2000.

[17] J. R. Guerci and J. S. Bergin, "Principal Components, Covariance Matrix Tapers, and the Subspace Teakage Problem," IEEE Trans. Aerospace and Elecronic Sys., pp. 152-162, Jan. 2002.

[18] I. C. Godara, "Application of Antenna Arrays to Mobile Communications, Part II: Beant-Forming and Direction-of-Arrival Considerations," Proceedings of the IEEE, pp. 1195-1245, August 1997.

[19] H. Krim and M. Viberg, "Two Decades of Array Signal Processing Research," IEEE Signal processing Magazine, pp. 67-94, July 1996.

[20] H. I. Van Trees, "Detection, Fstimation, and Modulation Theory, Par IV, Optimum Array Processing", John Wiley \& Sons, New York, 2002. 